\title{
Temporal and spatial dose distribution of radiation pneumonitis after concurrent radiochemotherapy in stage III non-small cell cancer patients
}

Mohammed Alharbi ${ }^{1}$, Stefan Janssen ${ }^{2,4}$, Heiko Golpon ${ }^{3}$, Michael Bremer ${ }^{1}$ and Christoph Henkenberens ${ }^{1 *}$ (D)

\begin{abstract}
Background and purpose: Radiation pneumonitis (RP) is the most common subacute side effect after concurrent chemoradiotherapy (CRT) for locally advanced non-small cell lung cancer. Several clinical and dose-volume (DV) parameters are associated with a distinct risk of symptomatic RP. The aim of this study was to assess the spatial dose distribution of the RP volume from first occurence to maximum volume expansion of RP.

Material and methods: Between 2007 and 2015, 732 patients with lung cancer were treated in an institution. Thirty-three patients met the following inclusion criteria: an RP grade II after CRT and a radiation dose $\geq 60$ Gy and no prior medical history of cardiopulmonary comorbidities. The images of the first chest computed tomography (CT) confirming the diagnosis of RP and the $\mathrm{CT}$ images showing the maximum expansion of RP were merged with the treatment plan. The RP volume was delineated within the treatment plan, and a DV analysis was performed to evaluate the lung dose volume areas in which the RP manifested over time and whether dose volume changes within the RP volume occurred.
\end{abstract}

Results: A change from clinical diagnosis to maximum expansion of RP was observed as the RP at clinical appearance mainly manifested in the lower dose areas of the lung, whereas the RP volume at maximum expansion manifested in the higher dose areas, resulting in a significant shift of the assessed relative mean dose volume proportions within the RP volume. The mean relative dose volume proportion 0- $\leq 20$ Gy decreased from $30.2 \%$ (range, $0-100$ ) to $21.9 \%$ (range, $0-100 ; p=0.04$ ) at the expense of the dose volume $>40$ Gy which increased from 39.2\% (range, 0-100) to 49. $8 \%$ (range, $0-100 ; p=0.02$ ), whereas the dose relative volume proportion $>20-\leq 40$ Gy showed no relevant change and slightly decreased from $30.6 \%$ (range, $0-85.7$ ) to $28.3 \%$, (range, $0-85.7 ; p=0.34$ ).

Conclusion: We observed a considerable increase in the relative dose proportions within the RP volume from diagnosis to maximum volume extent from low dose zones below $20 \mathrm{~Gy}$ to zones above $40 \mathrm{~Gy}$. Although the clinical impact on RP remains unknown, a reduction of healthy healthy lung tissue receiving >40 Gy (V40) might be an additional parameter for irradiation planning in lung cancer patients.

Keywords: Symptomatic radiation pneumonitis, Chemoradiotherapy, Lung cancer, Temporal dose volume change

\footnotetext{
* Correspondence: henkenberens.christoph@mh-hannover.de

'Department of Radio-Oncology, Medical School Hannover, Carl-Neuberg-Str.

1, 30625 Hannover, Germany

Full list of author information is available at the end of the article
} 


\section{Introduction}

Concurrent chemoradiotherapy (CRT) is the standard treatment for locally advanced non-small cell lung cancer (NSCLC), and radiation pneumonitis (RP) is one of the major dose-limiting side effects. High radiation doses significantly improve local control but also increase the incidence of severe RP and lead to poor quality of life and, in a few cases, death [1-3]. Several heterogeneous reports have investigated the incidence of clinically significant RP grade $\geq 2$ ranging from 10 to $30 \%$ after CRT with 60 to 70 Gray (Gy) and assessed numerous clinical and various dose volumetric (DV) parameters factors that are likely associated with clinically significant RP [4-9]. Since the introduction of three-dimensional (3D) treatment planning systems to the clinical routine, dose volume distributions of organs at risk can easily be assessed, and many studies have evaluated the association between DV parameters and the risk of RP $[5,7,8]$. Until now, no data assessing in which dose volume areas of the lung RP manifested at diagnosis and at maximum volume expansion have been available. Furthermore, it is unknown whether changes in relative dose volume proportions of the RP volume are related to volume changes.

Therefore, in the presented analysis, we assessed the spatial DV parameter changes in the RP volume at diagnosis and at maximum volume expansion over time.

\section{Material and methods}

\section{Patient parameters}

Between 2007 and 2015, 732 patients with lung cancer were treated in an institution and 33 of the 732 developed a RP grade II after CRT according to CTCAE v. 4.0 [10] and met the following inclusion criteria: no prior medical history of coronary heart disease requiring stent implantation or aortocoronary bypass surgery, known heart insufficiency, chronic obstructive pulmonary disease (COPD) or interstitial lung changes that are known to be associated with lung changes on lung imaging [11]. The patient and treatment characteristics are summarised in Table 1.

\section{CRT}

In all patients, radiotherapy was based on a planning CT (slice thickness: $3 \mathrm{~mm}$ ) and three-3D treatment planning using Oncentra MasterPlan ${ }^{\circledR}$ (Nucletron/Elekta, Stockholm, Sweden). Gross tumour volume (GTV) was identified by computed tomography, PET scan, and bronchoscopy. Hilar and mediastinal lymph nodes with a short axis $\geq 1.0 \mathrm{~cm}$ or pretreatment PET scan with standardised uptake values $(\mathrm{SUV})>3$ were included. The clinical target volume (CTV) was defined as GTV plus an individual margin up to $1.0 \mathrm{~cm}$ to account for microscopic tumour extension. For planning target volumes (PTVs), the CTVs were enlarged to allow for organ
Table 1 Patient characteristics

\begin{tabular}{ll}
\hline & Patients $(n=33)$ \\
\hline Median age (years, range) & $68.0(49-82)$ \\
Gender & \\
Male & $21(63.6 \%)$ \\
Female & $12(36.4 \%)$ \\
UICC-stage (TNM 7th ed.) & \\
IIIA & $14(42.4 \%)$ \\
IIIB & $19(57.6 \%)$ \\
Histological type & \\
Squamous cell carcinoma & $16(48.5 \%)$ \\
Adenocarcinoma & $17(51.5 \%)$ \\
Smoker status & \\
Non-smoker & $8(24.2 \%)$ \\
Smoker & $28(75.8 \%)$ \\
Chemotherapy & \\
CisplatinNinorelbine & $10(30.3 \%)$ \\
Carboplatin/Paclitaxel & $23(69.7 \%)$ \\
Localisation & $17.7(8.2-24.43)$ \\
Periphery & $25.1(12.0-45.01)$ \\
Central & $17.3(6.4-34.27)$ \\
Left & \\
Mean total lung dose (Gy) & $16(48.5 \%)$ \\
Mean V20 & \\
\hline & \\
\hline &
\end{tabular}

motion and setup variation and were expanded individually up to $1.0 \mathrm{~cm}$ in all directions. On-board cone-beam computed tomography was used at least weekly to confirm the correct patient positioning. Radiotherapy was carried out five times weekly at a daily single doses of 2.0 Gray (Gy). The radiation doses were 46-50.0 Gy to the mediastinum and 60-66 Gy to the primary tumour and pathological lymph nodes.

In general, irradiation was delivered using a conformal multifield technique with 6- to $10-\mathrm{MV}$ photons of a linac accelerator. Quantitative dose-volume analyses were performed using cumulative dose-volume histograms (DVH) to keep the mean lung dose (MLD) below 20 Gy and mean V20 and V30 below 30 and 20\%, respectively. The patients received either weekly concurrent carboplatin AUC2 plus paclitaxel $100 \mathrm{mg} / \mathrm{m}^{2}$ [12] or 2 cycles of cisplatin $20 \mathrm{mg} / \mathrm{m}^{2}$ day $1-4$ plus oral vinorelbine $50 \mathrm{mg} / \mathrm{m}^{2}$ on days 1,8 , and 15 [13]. None of the patients underwent induction or consolidation chemotherapy.

\section{RP diagnosis, treatment and follow-up}

The diagnosis of RP was based on the typical clinical symptoms such as new dyspnoea, non-productive cough, 
pleuritic chest pain and fever. A lung function test and a capillary blood gas test were also carried out. In all patients, computed tomography (CT) of the chest was performed within 3 days after presentation with RP symptoms to radiographically verify RP. CT scans of the chest were performed on a four-week basis until a substantial reduction in the pneumonitic ground glass opacities was observed. Thereafter, a CT scan of the chest and abdomen was routinely performed every 2 months. All patients underwent an oncological follow-up visit after the CT scan. Furthermore, the patients had biweekly clinical follow up visits until the steroids were tapered off completely $(0.5 \mathrm{mg} / \mathrm{kg}$ bodyweight prednisolone with at least $40 \mathrm{mg}$ initial dose per day). Every 5 days, the prednisolone dose was halved until the maintenance dose of $6 \mathrm{mg}$ per day was attained, which was usually maintained for an additional 6 weeks.

\section{DVH analysis for temporal and spatial dose distribution}

We reviewed all CT scans starting at the time point of first clinical occurrence of RP grade II until a significant reduction in pneumonitic areas was observed. We merged the images of the first chest CT that confirmed the diagnosis of RP after CRT and the CT images showing the maximum expansion of the typical pneumonitic ground glass opacities with the treatment plan using a planning treatment systems mutual algorithm. Am, JS, $\mathrm{BM}$ and $\mathrm{CH}$ delineated the pneumonitic areas using lung window of both $\mathrm{CT}$ scans at appearance and maximum expansion of RP lung changes (Fig. 1). In some cases the delineated volumes were modified on an individual basis to account for structural changes after CRT to avoid that former tumor areas after tumor shrinkage were incorrectly delineated as RP areas. A DVH analysis was then performed to assess in which dose areas of the lung the RP manifested at diagnosis and at maximum expansion to evaluate the relative dose volume proportions within the delineated RP volume at clinical appearance and at maximum expansion. The relative dose volume proportions of the patients were added to the relative mean dose volume proportions, which were used for statistical analysis.

\section{Statistical analysis}

Statistical analysis was performed using a commercially available software package (SPSS V. 24.0 for Windows, IBM, NY, USA). A paired samples t-test for parametric parameters was carried out to assess DV changes at diagnosis and at maximum RP expansion. Furthermore, we investigated whether clinical parameters, abnormal lung function test and capillary blood gas parameters below the normal values at RP diagnosis had a statistically significant influence on the DV changes within the
RP volume using a one-way repeated measures analysis of variance (rmANOVA).

\section{Results}

RP manifested clinically at median of 1.5 (range, 0.54.5) months after CRT, and the maximum volume expansion of the ground glass opacities on the CT images were observed at a median 3.75 (range, 2.0-7.75) months after CRT. All patients responded well to corticosteroids and none of the patients developed increased symptoms (e.g. requiring oxygen) after therapy with corticosteroids was initiated. The RP volume at diagnosis was at a median of $80.8 \mathrm{ccm}$ (range, 3.0-471.0) and increased significantly to $268.6 \mathrm{ccm}$ (range, 19.9-674.0; $\mathrm{p}=<0.001)$ at the time point of the greatest expansion after a median of 3.75 months after CRT. The mean dose within the pneumonitic lung areas was 33.4 Gy (range, 1.0-59.8) at diagnosis and was significantly higher with 37.8 Gy (range, 8.0-64.3; $p=0.026$ ) at the time of maximum expansion. Furthermore, the mean peak dose within the pneumonitic areas was significantly increased from initial appearance (52.3 Gy; range, 1.664.3) to maximum expansion (57.7 Gy; range, 8.0-66.32; $p=0.015)$. We observed that the RP at diagnosis mainly manifested in the lower dose areas of the lung, whereas the main proportion of the RP at maximum expansion was located in the higher dose areas and led to shifts in the relative dose distribution of the RP volume. We observed a statistically significant decrease in the relative mean dose volume proportion $0-\leq 20$ Gy from $30.2 \%$ (range, $0-100$ ) to 21.9 (range, $0-100 ; p=0.04$ ) and an increase $>40$ Gy from $39.2 \%$ (range, $0-100$ ) to $49.8 \%$ (range, $0-100 ; p=0.02$ ), whereas the relative mean dose volume proportion of $>20-\leq 40$ Gy showed a slight but not significant decrease from $30.6 \%$ (range, 0-85.7) to 28.3\%, (range, $0-85.7 ; p=0.34$ ). Considering the relative mean dose volume proportions below and above $30 \mathrm{~Gy}$, we also found a statistically significant increase in the relative dose volume proportions $>30$ Gy from $56.1 \%$ (range, $0-100$ ) to $66.1 \%$ (range, $0-100 ; p=0.03$ ). The results of the statistical analysis are shown in Table 2 and graphically in Fig. 2.

Regarding the results of the rmANOVA, none of the clinical parameters including side, localisation, sex, smoking status or chemotherapy regime had a statistically significant effect on the observed changes of the relative dose volume proportions of the RP volume. Patients with a vital capcity (VC) $<100 \%$ had a statistically significantly greater increase in the relative proportions $>30$ Gy $(p=0.039)$ and $>40$ Gy $(p=0.044)$, and a significantly smaller relative proportion $0-\leq 30$ Gy $(p=0.043)$ compared to patients with a normal VC $>100 \%$. None of the other lung function parameters or capillary blood gas concentrations had a significantly effect on the 


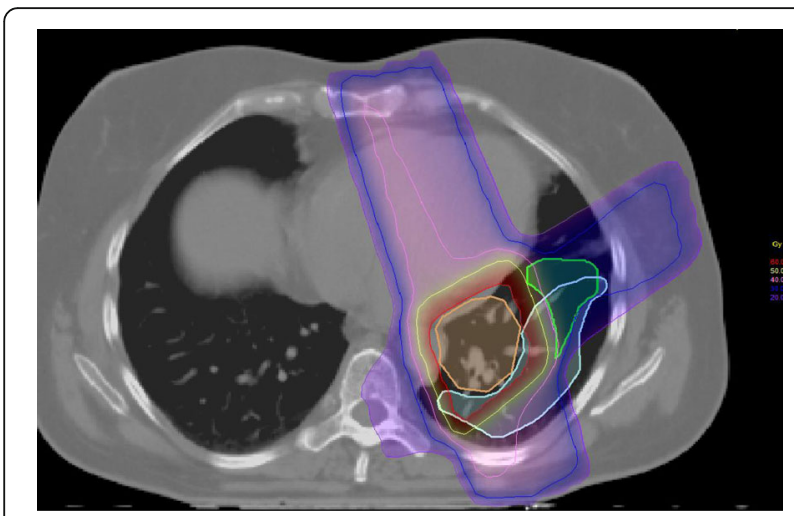

Fig. 1 Example for the delineation of the RP volume. Two CT scans of the chest to confirm the clinical diagnosis and showing the maximum expansion of RP were fused with the treatment plan and both the pneumonitic lung areas at the time point of clinical appearance and at maximum expansion were delineated. In this example, the RP at the time point of clinical appearance (green contour) was located outside the 50 Gy isodose (yellow), mainly in lung areas with doses below $30 \mathrm{~Gy}$. The larger RP volume at the time point of maximum expansion of RP (light blue contour) was also observed within the lung areas receiving more than $50 \mathrm{~Gy}$ (yellow 50 Gy isodose and red coloured the $60 \mathrm{~Gy}$ isodose) encompassing the bottom of the PTV (orange contour)

observed changes of the relative dose volume proportions within the RP volume. Furthermore patients treated with a photon energy $\geq 10$ megavoltage (MV) also had a significantly greater increase in the relative proportions $>40$ Gy $(p=0.033)$ compared to patients irradiated with $6 \mathrm{MV}$ only. The results of the rmANOVA are presented in Table 3.

\section{Discussion}

$\mathrm{RP}$ is the most common severe and clinically relevant subacute side effect after radiotherapy in lung cancer with a reported incidence of symptomatic RP of approximately $10-30 \%$. This range likely arises from different patient populations, a subjective RP scoring system and treatment-related factors such as radiotherapy technique and fractionation and chemotherapy [2, 7].

The application of oral steroids is recommended for treatment of symptomatic RP $\geq$ grade II. The therapy is not causal but merely symptomatic and does not influence the progression of RP to lung fibrosis. Several drugs, such as TNF-alpha or TGF-beta inhibitors, failed as causal treatments to interrupt inflammation progressing towards fibrosis, and none of these agents have been established as RP therapies [14]. Therefore, many studies have investigated patient- and treatment-related clinical parameters, such as comorbidities, concurrent chemotherapy and a variety of dose-volume parameters as risk factors for symptomatic RP [15-17]. All of these studies have suggested that certain clinical and dose volume parameters are associated with an increased risk or are even predictors of RP after radiotherapy. Thus far, to the best of our knowledge, no studies have reported changes in the dose areas of the lung when RP manifests with clinical symptoms or when RP had its greatest expansion. Therefore, there are no data on the associated changes of the relative dose volume proportions of the delineated RP areas from diagnosis to maximum expansion after definitive CRT for locally advanced NSCLC.

In the present analysis, we found that the delineated $\mathrm{RP}$ volume at clinical appearance was mainly located in lower dose areas of the lung, as the majority of the RP volume was formed by "low" mean dose volume proportions low dose volume, whereas the RP volume at maximum expansion was mainly located in the higher dose areas of the lung, resulting in shift for the mean relative dose proportions towards "high" dose volume proportions above $40 \mathrm{~Gy}$. The mean relative proportion of the intermediate dose volume interval from 20 to 40 Gy remained almost unchanged, suggesting that the clinical course of RP, until the decline of the typical RP signs on chest $\mathrm{CT}$ scans, was mainly determined by the high dose areas of the lung in the treatment plan. This was also confirmed to a lesser extent for the dichotomous discrimination of mean relative dose volume proportions $<30$ and $>30$ Gy.

These findings emphasise that in addition to the typical DVH parameters, such as V20 < 30\%, V30 < 20\% and an MLD <22 Gy [17], which are associated with an increased incidence of symptomatic RP after combinedmodality therapy for NSCLC, the volume of the lung receiving higher doses, in particular above 40 Gy (V40), should be taken into consideration and reduced as much as possible in irradiation planning. However, in some cases, the above-mentioned lung dose constraints have

Table 2 Proportions of the assessed relative dose proportions at diagnosis and at maximum radiographic expansion showing a statistically significant increase towards the higher dose areas $>30$ Gy and >40 Gy

\begin{tabular}{llll}
\hline Dose Interval (Gy) & Proportion (\%, range) at Diagnosis of RP & Proportion (\%, range) at maximum expansion of RP & $p$-Value \\
\hline $0-\leq 20$ & $30.2(0-100)$ & $21.9(0-100)$ & 0.04 \\
$>20-\leq 40$ & $30.6(0-86)$ & $28.3(0-78)$ & 0.34 \\
$>40$ & $39.2(0-100)$ & $49.8(0-95)$ & 0.02 \\
$0-\leq 30$ & $43.9(0-100)$ & $33.8(0-100)$ & 0.03 \\
$>30$ & $56.1(0-100)$ & $66.2(0-100)$ & 0.03 \\
\hline
\end{tabular}




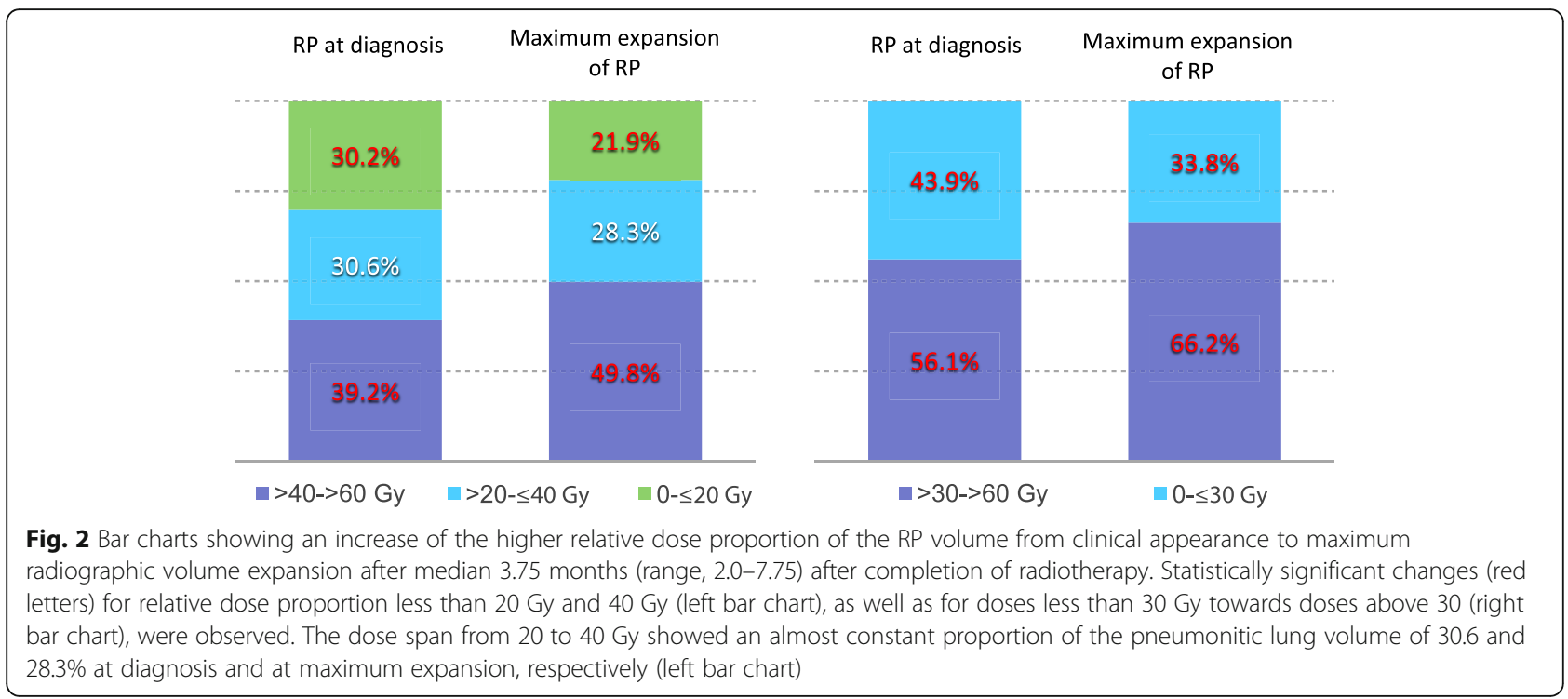

to be compromised to reach target volume coverage. Moreover, we do not know if smaller lung volumes receiving doses $>40$ Gy have either impact on the incidence of RP nor can we foreclose that this will reduce the RP volume, as this might be compensated by larger low dose-zones. Furthermore, the impact on the clinical symptoms is ambiguous, because the relative lower dose proportions $0-\leq 20$ Gy and $>20-\leq 40$ Gy within the RP volume at clinical occurrence have been 30.2 and 30.6\%, respectively. This might imply that the lung volumes $>40$ Gy may had no significant influence on symptoms at clinical appearance of RP.

Brierie et al. who showed that RP depends on sparing of total lung volumes at 40 Gy resulted in a significantly decreased incidence of RP 6 months after radiotherapy in a cohort of 579 NSCLC patients of which 449 (77.5\%) had concurrent chemotherapy and a radiotherapy dose of at least 50 Gy [18]. Their results and our findings in the present analysis suggest that in patients with locally advanced NSCLC (UICC stage III) receiving CRT, the radiation oncologist should monitor the dose volume of the healthy lung tissue receiving $40 \mathrm{~Gy}$ and above because this is likely to have an impact on both the incidence and the volumetric expansion of symptomatic RP, and sparing the lung treatment with $>40$ Gy (V40) might be beneficial.

In addition to the above-mentioned classical dose constraints, several static and functional parameters are proposed to reduce the risk of RP.

Other factors include the V5 [19], the lung volume receiving a dose less than 5 Gy below $1500 \mathrm{ccm}$ [16], the ipsilateral V20 and ipsilateral V30 [17], and functional

Table 3 Results of the one-way repeated measures analysis of variance (rmANOVA) of the assessed clinical, lung function and blood gas parameters at diagnosis of RP parameters

\begin{tabular}{|c|c|c|c|c|c|c|c|c|c|c|c|c|c|}
\hline & $\begin{array}{l}\text { Side (left } \\
\text { / right) }\end{array}$ & $\begin{array}{l}\text { Localisation } \\
\text { (central } \\
\text { /peripheral) }\end{array}$ & $\begin{array}{l}\text { Smoking } \\
\text { status } \\
\text { (smoker / } \\
\text { non- } \\
\text { smoker) }\end{array}$ & Sex & $\begin{array}{l}\text { Chemotherapy (Cis- } \\
\text { platin+Vinorelbine / } \\
\text { Carboplatin + } \\
\text { Paclitaxel) }\end{array}$ & $\begin{array}{l}\text { Energy } \\
\geq 10 \mathrm{MV}\end{array}$ & $\begin{array}{l}\text { FEV1 } \\
<80 \%\end{array}$ & $\begin{array}{l}\text { VC } \\
<100 \%\end{array}$ & $\begin{array}{l}\mathrm{pO}_{2} \\
<70 \%\end{array}$ & $\begin{array}{l}\mathrm{pCO}_{2} \\
>42 \%\end{array}$ & $\begin{array}{l}\text { DLCO } \\
<70 \%\end{array}$ & $\begin{array}{l}\text { RAW >0.35 } \\
\mathrm{kPa} / \mathrm{L} / \mathrm{s}\end{array}$ & $\begin{array}{l}\mathrm{HbCO} \\
>0.5 \%\end{array}$ \\
\hline $0-\leq 20$ & 0.59 & 0.61 & 0.26 & 0.87 & 0.58 & 0.30 & 0.66 & 0.12 & 0.33 & 0.35 & 0.16 & 0.50 & 0.14 \\
\hline$>20-40$ & 0.62 & 0.95 & 0.08 & 0.22 & 0.82 & 0.30 & 0.91 & 0.26 & 0.17 & 0.66 & 0.24 & 0.57 & 0.65 \\
\hline$>40$ & 0.66 & 0.70 & 0.90 & 0.38 & 0.23 & 0.033 & 0.43 & 0.044 & 0.94 & 0.21 & 0.06 & 0.60 & 0.20 \\
\hline $0-\leq 30$ & 0.47 & 0.72 & 0.63 & 0.41 & 0.19 & 0.10 & 0.43 & 0.043 & 0.46 & 0.21 & 0.07 & 0.60 & 0.17 \\
\hline$>30$ & 0.44 & 0.66 & 0.67 & 0.36 & 0.19 & 0.10 & 0.42 & 0.039 & 0.49 & 0.20 & 0.07 & 0.61 & 0.08 \\
\hline
\end{tabular}

Statistically significant results are highlighted with a green background

Abbreviation: $M V$ Megavoltage, $F E V 1$ forced expiratory volume in $1 \mathrm{~s} ; V C$ vital capacity, $p \mathrm{O}_{2}$ partial pressure of oxygen, $p C \mathrm{O}_{2}$ partial pressure of carbon dioxide, $D L C O$ diffusion capacity of carbon monoxide, RAW airway resistance, $\mathrm{HbCO}$ fraction of carboxyhaemoglobin 
differences in regional lung perfusion assessed by single photon emission CT lung perfusion scans [20], which are less common and were not used for irradiation planning for our patients. However, these parameters might have had an influence on the observed changes in the relative dose volume proportions of the RP volume from diagnosis to the maximum expansion RP. However, many these dosimetric variables tend to be very collinear, and therefore, differences among different dosimetric variables may be small leading to an unknown merit of decreasing one (e.g., lowering V20) at the expense of another (e.g., raising the V5) [21].

The limitations of our study include the inherent weaknesses of its retrospective nature. In addition, the relatively small patient sample size $(n=33)$ might have introduced statistical bias in this report. Nevertheless, the results seem to be robust due to the homogenous patient cohort, which included only medically fit patients with NSCLC treated with definitive CRT. Otherwise, no conclusion could be drawn for patients who had either mediastinal radiotherapy post lung surgery or sequential chemotherapy. The intention was to reduce possible confounders for the delineation of the ground-glass opacities of RP. Thus, we excluded patients with severe comorbidities, such as COPD or interstitial lung diseases, which may lead to an estimated misdiagnosis rate of up to $20 \%$ of RP [22]. This might be a reason why typical lung function parameters associated with symptomatic RP, such as decreased forced expiratory volume in $1 \mathrm{~s}$ (FEV1) and diffusion capacity for carbon monoxide (DLCO) $[23,24]$, showed no significant effect on the observed shift from low to high dose relative volume proportions, whereas our statistical analysis using rmANOVA supported the assumption that a vital capacity (VC) $<100 \%$ at diagnosis of RP might be an indicator for a relevant shift. Recently, Bradley et al. demonstrated that patients of the RTOG 0617 trial treated with intensity-modulated radiation therapy (IMRT) had symptomatic RP less often than patients treated with 3D-conformal CRT due to a significantly lower V20, and in general, IMRT allows for better protection of healthy lung tissue than 3D-RT [25]. Due to our results in which the majority of the RP volume at maximum expansion was located in lung areas with higher doses above $20 \mathrm{~Gy}$, we also recommend IMRT for CRT in patients with NSCLC.

\section{Conclusion}

We observed a considerable increase in the relative dose proportions within the RP volume from diagnosis to maximum volume extent from low dose zones below 20 Gy to zones above 40 Gy. The impact on incidence and severity of RP is still unknown. More clinical data are needed to assess the impact of these finding on the incidence and severity of RP. Nevertheless the volume of healthy lung tissue receiving $>40$ Gy (V40) might be an additional parameter for irradiation planning in lung cancer patients.

\section{Acknowledgements \\ None.}

Funding

No funding.

Availability of data and materials

The complete statistical dataset used for the current study are available from the corresponding author on reasonable request.

\section{Authors' contributions}

AM, JS and $\mathrm{CH}$ collected and analysed the data and wrote the manuscript. $\mathrm{GH}$ treated the patients after pneumonitis occurred and provided patients' data. BM was a major contributor in writing the manuscript. All authors read and approved the final manuscript.

Ethics approval and consent to participate

Ethics approval and consent to participate was waived due to the retrospective character of the study.

Consent for publication

Not applicable.

\section{Competing interests}

The authors declare that they have no competing interests.

\section{Publisher's Note}

Springer Nature remains neutral with regard to jurisdictional claims in published maps and institutional affiliations.

\section{Author details}

${ }^{1}$ Department of Radio-Oncology, Medical School Hannover, Carl-Neuberg-Str. 1, 30625 Hannover, Germany. ${ }^{2}$ Joint Practice Radiooncology Hannover, Rundestr. 10, 30161 Hannover, Germany. ${ }^{3}$ Department of Pneumology, Medical School Hannover, Carl-Neuberg-Str. 1, 30625 Hannover, Germany. ${ }^{4}$ Department of Radiation Oncology, University of Lübeck, Ratzeburger Ave. 160, 23562 Lübeck, Germany.

Received: 23 August 2017 Accepted: 3 October 2017

Published online: 02 November 2017

\section{References}

1. Bradley JD, Paulus R, Komaki R, Masters G, Blumenschein G, Schild S, et al. Standard-dose versus high-dose conformal radiotherapy with concurrent and consolidation carboplatin plus paclitaxel with or without cetuximab for patients with stage IIIA or IIIB non-small-cell lung cancer (RTOG 0617): a randomised, two-by-two factorial phase 3 study. Lancet Oncol. 2015;16:187-99.

2. Schröder C, Engenhart-Cabilic R, Vorwerk H, Schmidt M, Huhnt W, Blank E, et al. Changes in pulmonary function and influencing factors after high-dose intrathoracic radio(chemo)therapy. Strahlenther Onkol. 2017;193:125-31.

3. Schröder C, Engenhart-Cabilic R, Vorwerk H, Schmidt M, Huhnt W, Blank E, et al. Patient's quality of life after high-dose radiation therapy for thoracic carcinomas : changes over time and influence on clinical outcome. Strahlenther Onkol. 2017;193:132-40.

4. Borst GR, De Jaeger K, Belderbos JS, Burgers SA, Lebesque JV. Pulmonary function changes after radiotherapy in non-small-cell lung cancer patients with long-term disease-free survival. Int J Radiat Oncol Biol Phys. 2005;62:639-44

5. Claude L, Perol D, Ginestet C, Falchero L, Arpin D, Vincent M, et al. A prospective study on radiation pneumonitis following conformal radiation therapy in non-small-cell lung cancer: clinical and dosimetric factors analysis. Radiother Oncol. 2004;71:175-81.

6. Henkenberens C, Janssen S, Lavae-Mokhtari M, Leni K, Meyer A, Christiansen $\mathrm{H}$, et al. Inhalative steroids as an individual treatment in symptomatic lung cancer patients with radiation pneumonitis grade $\|$ after radiotherapy - a 
single-centre experience. Radiat Oncol. 2016; https://doi.org/10.1186/ s13014-016-0580-3.

7. Mao J, Kocak Z, Zhou S, Garst J, Evans ES, Zhang J, et al. The impact of induction chemotherapy and the associated tumor response and subsequent radiation-related changes in lung function and tumor response. In J Radiat Oncol Biol Phys. 2007;67:1360-9.

8. Shi A, Zhu G, Wu H, Yu R, Li F, Xu B. Analysis of clinical and dosimetric factors associated with severe acute radiation pneumonitis in patients with locally advanced non-small cell lung cancer treated with concurrent chemotherapy and intensity-modulated radiotherapy. Radiat Oncol. 2010; https://doi.org/10.1186/1748-717X-5-35.

9. Tsujino K, Hirota S, Endo M, Obayashi K, Kotani Y, Satouchi M, et al. Predictive value of dose-volume histogram parameters for predicting radiation pneumonitis after concurrent chemoradiation for lung cancer. Int J Radiat Oncol Biol Phys. 2003;55:110-5.

10. National Cancer Institute. Division of Cancer Treatment \& Diagnosis. https:// ctep.cancer.gov/protocolDevelopment/electronic_applications/docs/ Reverse_Mapping_CTCAE_4_to_CTCAE_3.xls. Accessed 14 March 2017.

11. Zhang XJ, Sun JG, Sun J, Ming H, Wang XX, Wu L, et al. Prediction of radiation pneumonitis in lung cancer patients: a systematic review. J Cancer Res Clin Oncol. 2012:138:2010-6.

12. Belani CP, Choy H, Bonomi P, Scott C, Travis P, Haluschak J, et al. Combined chemoradiotherapy regimens of paclitaxel and carboplatin for locally advanced non-small-cell lung cancer: a randomized phase II locally advanced multi-modality protocol. J Clin Oncol. 2005;23:5883-91.

13. Flentje M, Huber RM, Engel-Riedel W, Andreas S, Kollmeier J, Staar S, et al. GILT-A randomised phase III study of oral vinorelbine and cisplatin with concomitant radiotherapy followed by either consolidation therapy with oral vinorelbine and cisplatin or best supportive care alone in stage III nonsmall cell lung cancer. Strahlenther Onkol. 2016;192:216-22.

14. Tsoutsou PG. The interplay between radiation and the immune system in the field of post-radical pneumonitis and fibrosis and why it is important to understand it. Expert Opin Pharmacother. 2014;15:1781-3.

15. Parashar B, Edwards A, Mehta R, Pasmantier M, Wernicke AG, Sabbas A, et al. Chemotherapy significantly increases the risk of radiation pneumonitis in radiation therapy of advanced lung cancer. Am J Clin Oncol. 2011;34:160-4.

16. Tsujino K, Hashimoto T, Shimada T, Yoden E, Fujii O, Ota Y, et al. Combined analysis of V20, VS5, pulmonary fibrosis score on baseline computed tomography, and patient age improves prediction of severe radiation pneumonitis after concurrent chemoradiotherapy for locally advanced nonsmall-cell lung cancer. J Thorac Oncol. 2014;9:883-90.

17. Ramella S, Trodella L, Mineo TC, Pompeo E, Stimato G, Valentini V, et al. Adding ipsilateral V20 and V30 to conventional dosimetric constraints predicts radiation pneumonitis in stage IIIA-B NSCLC treated with combined-modality therapy. Int J Radiat Oncol Biol Phys. 2010;76:110-5.

18. Briere TM, Krafft S, Liao Z, Martel MK. Lung size and the risk of radiation Pneumonitis. Int J Radiat Oncol Phys. 2016;94:377-84.

19. Khalil AA, Hoffmann L, Moeller DS, Farr KP, Knapp MM. New dose constraint reduces radiation-induced fatal pneumonitis in locally advanced non-small cell lung cancer patients treated with intensity-modulated radiotherapy. Acta Oncol. 2015;54:1343-9.

20. Farr KP, Kallehauge JF, Moller DS, Khalil AA, Kramer S, Bluhme H, et al. Inclusion of functional information from perfusion SPECT improves predictive value of dose-volume parameters in lung toxicity outcome after radiotherapy for non-small cell lung cancer: a prospective study. Radiother Oncol. 2016;117:9-16.

21. Palma DA, Senan S, Tsujino K, Barriger RB, Moreno M, Bradley JD, et al. Predicting radiation pneumonitis after chemoradiation therapy for lung cancer: an international individual patient data meta-analysis. Int J Radiat Oncol Biol Phys. 2013;85:444-50.

22. Kocak Z, Evans ES, Zhou SM, Miller KL, Folz RJ, Shafman TD, et al. Challenges in defining radiation pneumonitis in patients with cancer lung cancer. Int J Radiat Oncol Biol Phys. 2005;62:635-8.

23. Wang D, Zhu J, Sun J, Li B, Wang Z, Wei L, et al. Functional and biologic metrics for predicting radiation pneumonitis in locally advanced non-small cell lung cancer patients treated with chemoradiotherapy. Clin Transl Oncol. 2012;14:943-52.

24. Lopez Guerra JL, Gomez D, Zhuang Y, Levy LB, Eapen G, Liu H, et al. Change in diffusing capacity after radiation as an objective measure for grading radiation pneumonitis in patients treated for non-small-cell lung cancer. Int J Radiat Oncol Biol Phys. 2012;83:1573-9.
25. Chun SG, Hu C, Komaki RU, Timmermann RD, Schild SE, Bogart JA, et al. Impact of intensity-modulated radiation therapy technique for Lacally advanced non-Sall-cell lung cancer: a secondary analysis of the NRG oncology RT 0617 randomized clinical trial. J Clin Oncol. 2017;35:56-62.

\section{Submit your next manuscript to BioMed Central and we will help you at every step:}

- We accept pre-submission inquiries

- Our selector tool helps you to find the most relevant journal

- We provide round the clock customer support

- Convenient online submission

- Thorough peer review

- Inclusion in PubMed and all major indexing services

- Maximum visibility for your research

Submit your manuscript at www.biomedcentral.com/submit
Biomed Central 